\title{
The time machine: structure-based elucidation of timekeeping mechanisms by the cyanobacterial circadian clock
}

\author{
Carrie L. Partch ${ }^{1,2}$, Jeffrey Swan ${ }^{1}$, Joel Heisler ${ }^{3}$, Susan S. Golden ${ }^{2,5}$, Andy \\ LiWang $2,3,6,7,8$ \\ ${ }^{1}$ Department of Chemistry \& Biochemistry, University of California Santa Cruz \\ ${ }^{2}$ Center for Circadian Biology, University of California San Diego \\ ${ }^{3}$ Chemistry \& Chemical Biology, University of California Merced \\ ${ }^{5}$ Division of Biological Sciences, University of California San Diego \\ ${ }^{6}$ Quantitative \& Systems Biology, University of California Merced \\ ${ }^{7}$ School of Natural Sciences, University of California Merced \\ ${ }^{8}$ Health Sciences Research Institute, University of California Merced
}

Circadian rhythms are an ancient evolutionary adaptation found across the domains of life, synchronizing behavior and physiology to the daily solar cycle for optimal fitness. In cyanobacteria, timekeeping is established by three Kai proteins (KaiA, B and C) that form distinct protein complexes throughout the day, comprising a molecular clock that measures $\sim 24$-hour timing. Remarkably, this protein-based clock exhibits accurate, temperaturecompensated timekeeping in the presence of only ATP in vitro. The LiWang lab recently discovered that KaiB undergoes a rare transition from its tetrameric ground state to a rare fold-switched, monomeric signaling state that is needed to bind KaiC and advance the clock in its evening phase ${ }^{1}$. To address the structural basis for this nighttime complex, we locked KaiB into its fold-switched state, allowing us to solve crystal and NMR structures of four key complexes that demonstrate how KaiB docks cooperatively on the 360 kDa KaiC hexamer to recruit KaiA and the output protein CikA2. A new structure of the output protein SasA bound to KaiC illustrates how KaiB uses structural mimicry to compete with SasA binding at dusk. Collectively, these structures reveal how protein interactions regulate day/night transitions and highlight the essential role of fold-switching by KaiB for control of timekeeping and output signaling in the cyanobacterial circadian clock.

${ }^{1}$ Chang, Y.G., Cohen, S.E., Phong, C., Myers, W.K., Kim, Y.I., Tseng, R., Lin, J., Zhang, L., Boyd, J.S., Lee, Y., Kang, S., Lee, D., Li, S., Britt, R.D., Rust, M.J., Golden, S.S., LiWang, A. (2015) Circadian Rhythms. A protein fold switch joins the circadian oscillator to clock output in cyanobacteria. Science 349:324-8.

${ }^{2}$ Tseng, R. ${ }^{*}$, Goularte, N.F. ${ }^{*}$, Chavan, A. ${ }^{*}$, Luu, J., Cohen, S.E., Chang, Y.G., Heisler, J., Li, S, Michael, A.K., Tripathi, S., Golden, S.S., LiWang, A., Partch, C.L. (2017) Structural basis of the day-night transition in a bacterial circadian clock. Science, 355:1174-1180. 